\title{
Movable-bed experiments using spur dike to concentrate flow in one channel of multithreaded channel model
}

\begin{abstract}
This paper presents the results of an experimental study in which an acoustic Doppler velocimeter (ADV) was used to monitor the longitudinal velocity profiles and discharge proportions in a channel with a mobile bed and banks. The resultant channel bed elevations were also monitored. The physical model consisted of a channel with two branches and a midchannel bar. A spur dike was located at the upstream of the branches. The main variable parameters in this study were the length of the midchannel bar and the length of the spur dike. These variables were investigated by performing nine trials (with differing spur dike and channel bar lengths) with different corresponding discharges. Clear water conditions were maintained in the channel throughout this study. The results showed that the spur dike diverted and redistributed the discharge between the branches of the multithreaded channel. The highest discharge proportion was obtained when the spur dike's length was one-third of the main channel's width and when the midchannel bar's length was short. In addition, the slope of the channel's bed was steeper in the branch that carried the highest discharge proportion.
\end{abstract}

Keyword: Midchannel bar; Spur dike; Discharge proportion; Longitudinal velocity profile 